\title{
TEMPO DE REAÇÃO MOTORA NO VOLEIBOL
}

\author{
Anderson Pontes Morales \\ Mestre em Ciência da Motricidade Humana/Laboratório de Biociências da Motricidade \\ Humana/UCB-RJ \\ andersonmrl@hotmail.com
}

Ronaldo Nascimento Maciel

Mestre em Ciência da Motricidade Humana/Laboratório de Biociências da Motricidade Humana/UCB-RJ

rnmvolei@hotmail.com

\section{Vernon Furtado da Silva}

Doutor em Philosophy/University Of Maryland/U.M. College Park, Maryland/USA

vernonfurtado2005@yahoo.com.br

\section{Alair Pedro Ribeiro de Souza e Silva}

Doutor em Educação Física/University Of Maryland/U.M. College Park, Maryland/USA

ribeirops@uol.com.br

\section{RESUMO}

Enquanto jogo desportivo coletivo, o voleibol está estruturado por normas ou regras específicas, que determinam a conduta a ser tomada pelos participantes e expressam a lógica e característica do jogo. Por ser um desporto com características "abertas", ou seja, pouco previsíveis, o desempenho motor está diretamente relacionado às capacidades de se prever e responder às mudanças acontecidas no ambiente. Assim, $\mathrm{O}$ tempo de reação motora torna-se um importante aspecto para o sucesso de atletas envolvidos na modalidade voleibol. O objetivo do estudo é de analisar criticamente os conceitos do tempo de reação no voleibol. Para preparar uma saída motora apropriada, o organismo tem que ser eficiente na codificação de estímulos relevantes, tendo o tempo de reação motor como a principal fator no desempenho dos atletas. Em razão da velocidade característica do voleibol durante as ações do jogo, aliada a imprevisibilidade do ambiente, busca-se desenvolver capacidades como a percepção, a antecipação e a diferenciação das situações práticas pelo atleta, com esses aspectos tendo uma importância por vezes superior à técnica requerida para a execução do fundamento.

Palavras-chave: Voleibol; Tempo de Reação Motor; Processamento de Informações.

\begin{abstract}
While playing collective sports, volleyball is structured by specific policy or rules, which determine the action to be taken by the participants and expressed the logic and character of the game. Because it is a sport with features "open", or a little predictable, the motor performance is directly related to the capabilities to predict and respond to changes taking place in the environment. Thus, the motor reaction time becomes an important aspect for the success of athletes involved in the sport volleyball. The objective is to critically analyze the concepts of reaction time in volleyball. To prepare an appropriate behavioral output, the body has to be efficient in the encoding of relevant stimuli, and motor reaction time as the main factor in the performance of athletes. Due to the characteristic velocity during the volleyball game actions, coupled with the unpredictability of the environment, we seek to develop skills such as perception, anticipation and differentiation of the athlete practical situations, with these important aspects of having a sometimes superior to the technique required for the execution of the foundation.
\end{abstract}

Keywords: Volleyball; Motor Reaction Time; Information Processing. 


\section{INTRODUÇÃO}

Nos últimos anos na área da aprendizagem motora, tem havido uma preocupação, na elaboração de uma série de princípios e abordagens teóricas, que têm subservido ao ensino hábilmotor relacionado às várias modalidades esportivas de natureza individuais ou coletivas. $\mathrm{O}$ voleibol desde a sua criação em 1895, por Willian G. Morgan, vem evoluindo em seus planos de treinamentos, fazendo com que a modalidade seja fonte de numerosos estudos, que visam potencializar o rendimento dos atletas e onde detalhes encontrados no âmbito técnico, tático e físico tornam-se diferenciais no resultado final alcançado por uma equipe em competição, transformando-se desta forma em um jogo altamente competitivo (BIZZOCHI, 2004; PRUDENCIO \& TUMELERO, 2006; WAGNER \& SOUZA, 2007).

Por ser um desporto com características "abertas", ou seja, pouco previsíveis, o desempenho motor está diretamente relacionado às capacidades de se prever e responder às alterações acontecidas no ambiente (BARCELOS et al., 2009). Para identificar estas ações, é necessário que o entendimento sobre as nuances táticas do jogo sejam treinadas e que esses treinos requeiram do atleta um alto grau de conhecimento e percepção sobre os eventos de desempenho relacionados ao desporto, já que o voleibol é caracterizado por ações de períodos curtos, intensidade máxima e intervalos reduzidos (KRAMER, 2004; PRUDENCIO \& TUMELERO, 2006).

Neste escopo, o tempo de reação torna se um importante aspecto para o sucesso de atletas envolvidos na modalidade. O tempo de reação motora é umas das medidas de resultado de comportamento mental/motor mais utilizadas em pesquisa, podendo influenciar o resultado ou efeitos da execução de uma habilidade motora, além de ser decisivo para a melhora do desempenho. Para Santos \& Tani (1995) o tempo de reação, está interligado ao tempo de processamento de informações, necessário para uma execução de uma resposta, que, por sua vez, permite fazer inferências sobre os mecanismos subjacentes que envolvem tal tarefa em termos de desempenho; quanto menor for o tempo de reação maior a eficiência dos mecanismos e processos centrais.

Sendo assim, este artigo se caracteriza como de revisão de literatura, tendo como objetivo analisar criticamente os conceitos vigentes acerca do tema em questão. Para este trabalho de pesquisa, do tipo analítica, de revisão, foram pesquisados artigos indexados nas principais bases de dados disponíveis, utilizando-se no campo "palavras de títulos" os termos voleibol, aprendizagem e tempo de reação. Foram ainda usados alguns dos principais livros textos que discorrem sobre as áreas acima mencionadas.

\section{REVISÃO DE LITERATURA}

\subsection{Tempo de Reação Motora: bases e conceitos}

O tempo de reação pode ser definido como o intervalo existente entre a apresentação de um estímulo não antecipado e o início da resposta. Está presente como importante variável em vários esportes, além de representar uma parte real de diversas atividades do nosso dia-a-dia (SCHMIDT e WRISBERG, 2001). Alguns autores identificam duas fases para sua execução, a pré-motora e a motora (SILVA, 2006; MIYAMOTO e MEIRA Jr. 2004). O Tempo de reação é um indicativo da eficácia da velocidade de processamento de informações dos mecanismos centrais, representando o padrão de coordenação neuromuscular onde os estímulos são decodificados pelo corpo através de diferentes processos químicos, físicos e mecânicos, e iniciar a execução das ações motoras mais adequadas para a resposta (VAGHETTI, ROESLER e ANDRADE, 2007; WEINECK, 2003). Brown (2004) afirma que o tempo de reação é uma função complexa que inclui componentes mentais, físicos, inatos e treináveis, havendo diferenças individuais entre os indivíduos, mas com todos podendo melhorar o seu desempenho. 
O tempo de reação é basicamente dividido em: Tempo de reação simples, onde apenas um estímulo ocorrerá, com o indivíduo devendo responder prontamente a este, Tempo de reação de escolha, onde dois ou mais estímulos aparecerão e o indivíduo deverá identificá-los, e, a seguir, selecionar a resposta mais adequada para cada estímulo, dentre as várias respostas possíveis, Tempo de reação de discriminação, que é o intervalo de tempo entre a aparição de um dos vários estímulos possíveis e o começo da única resposta. No tempo de reação de escolha observamos a famosa lei de Hick, que descreve uma relação estável entre o número de alternativas de estímulos e o tempo de reação, que tende a ser maior quanto maior for o número de estímulos (SCHIMIDT e WRISBERG, 2001).

Em relação ao tempo de reação simples, pode-se afirmar que é o tempo de reação mais breve que um indivíduo é capaz de conseguir. Isto se deve a menor participação dos processos de tomada de decisão. De acordo com Chagas et al. (2005), uma pessoa que tem um bom tempo de reação simples pode ter uma influência positiva no tempo de reação de escolha. O tempo de reação simples também se caracteriza pela alta influência genética, sendo menos treinável do que o tempo de reação de escolha (MIYAMOTO e MEIRA Jr., 2004).

Segundo Weineck (2003), O tempo de reação difere para estímulos acústicos, óticos e táteis, tanto em indivíduos treinados, quanto nos não treinados. A conversão das ondas luminosas em impulsos neuronais é mais demorada do que a transformação das ondas sonoras, devido aos processos acústicos serem mais diretos do que os visuais. Quando a resposta envolve a combinação de dois ou mais fatores (visual e manual, por exemplo), o tempo de reação tende a ser mais longo (KOKUBU e ANDO, 2006). O gênero também representa fator que causa diferença, com os homens sendo, de forma geral, mais rápidos. A idade é outra variável a se considerar. Os indivíduos que adquirem uma bagagem motora rica na infância tendem a ter um melhor tempo de reação, com o desempenho máximo sendo alcançado entre 20 e 30 anos, sendo reduzido a partir daí (WEINECK, 2003). O mesmo autor (2003) afirma que as respostas para atividades com as mãos são mais rápidas do que as com os pés, e que ambas são mais rápidas do que quando a resposta envolve simultaneamente pés e mãos. Portela (2005) classifica os fatores que influenciam o tempo de reação como definitivos, que são a idade, deficiência física e gênero, temporários, grupo em que estão as enfermidades, o estado emocional, sono e drogas, e condicionados, onde estaria o número de alternativas estímulo-resposta e sua compatibilidade.

\subsection{Voleibol e o Tempo de Reação Motora}

Enquanto jogo desportivo coletivo, o voleibol está estruturado por normas ou regras específicas, que determinam a conduta a ser tomada pelos participantes e expressam a lógica e característica do jogo. $\mathrm{O}$ voleibol pode ser definido como um desporto situacional, por requerer grande capacidade de adaptação a situações que se modificam continuamente e em curto tempo. $\mathrm{O}$ movimento para o voleibolista é uma ação motora em resposta aos estímulos que acontecem no ambiente de jogo (MACHADO, 2006). De acordo com Stanganelli et al. (2006), é uma modalidade complexa que requer do praticante uma combinação de capacidades motoras coordenativas e cognitivas, sendo que quanto mais alto o nível do jogo, maior é a exigência na utilização dessas capacidades.

Por ser um desporto com características "abertas", ou seja, pouco previsíveis, o desempenho motor está diretamente relacionado às capacidades de se prever e responder às mudanças acontecidas no ambiente (FONTANI et al., 2006; ROCHA, 2005; FONTANI et al., 1999). Maggil (2000) define uma habilidade esportiva como "aberta" quando a mesma envolve um ambiente imprevisível e em contínua mudança. A capacidade de reagir prontamente a um estímulo é vital na obtenção do sucesso de um atleta em desportos com características como as do voleibol. Para preparar uma saída motora apropriada, o organismo tem que ser eficiente na codificação de estímulos relevantes (VOLCHAN et al., 2003). 
O tempo de reação é resultante de três passos: primeiro o indivíduo percebe o estímulo; a seguir ele irá selecionar a resposta que julgar mais conveniente de acordo com a situação; efetuando finalmente a resposta (SILVA et al., 2003; SCHMIDT e WRISBERG, 2001). Qualquer fator que prolongue um desses estágios durante todo o processo irá interferir negativamente no tempo de reação (ANDRADE, 2004).

A necessidade de tomar decisões rápidas no menor tempo possível é difícil para qualquer atleta, independente do seu nível. O tempo de reação é uma capacidade motora coordenativa que vem ganhando destaque no campo da pesquisa sobre respostas motoras, sendo atualmente uma das variáveis intervenientes ao desempenho mais pesquisadas, devido a sua importância nos mais variados eventos desportivos (BOMPA, 2005; BARBANTI, 1996).

Na prática desportiva, especificamente no voleibol, o tempo de reação está inevitavelmente atrelado a outras capacidades, como por exemplo, a atenção e a memória, pois devido ao cenário de alta imprevisibilidade característico da maioria das ações do jogo, o atleta tem que ser seletivo em focar aquilo que é mais importante naquele momento, sendo, portanto, uma variável que antecede a execução do movimento (OLIVEIRA, OLIVEIRA e SOUZA, 2006). Estratégias, como a utilização de pontos denominados de fixação e/ou de atenção, devem ser incentivadas, de forma que sirvam como elementos que possam manter a atenção, facilitando a posterior tomada de decisão (MACHADOPINHEIRO, GAWRYSZEWSKI e RIBEIRO DO VALLE, 1998). Como são situações extremamente rápidas, não há possibilidade de ter um feedback sobre erros cometidos e corrigi-los durante a execução (SCHIMIDT e WRISBERG, 2001). Desempenhos excelentes por vezes são alcançados pela capacidade de antecipação de atletas de alto rendimento, que intencionalmente provocam um desequilíbrio antes da ação do adversário, através da posição de expectativa, que facilita a partida em direção a bola (ARAÚJO, 1994). A velocidade com a qual atleta consegue detectar características do ambiente está diretamente relacionada ao sucesso da ação motora (PORTELA, 2005).

Desta forma, o Treinamento se constitui em uma importante ferramenta para o desenvolvimento do Tempo de reação. As habilidades inerentes ao desporto devem ser treinadas através de atividades que proporcionem desafios que estimulem a capacidade cognitiva. Quanto melhor for a capacidade de análise da situação de jogo, mais rápida pode ser a preparação da resposta adequada (SILVA, 2006). Andrade (2004) afirma que em jogos como o voleibol o atleta deve ser capaz de organizar suas ações a fim de atingir os objetivos propostos, tornando-se fundamental o desenvolvimento do seu raciocínio tático. Dentre a gama de exercícios que podem ser utilizados, devem ser priorizados aqueles que possuem uma maior semelhança com o gesto esportivo. Desta forma, estaremos atendendo ao princípio da especificidade do treinamento desportivo e temos a possibilidade de conseguir uma maior transferência do trabalho físico para as situações de jogo (BOSSI, 2008). Silva (2006) afirma que a percepção de um determinado estímulo depende diretamente do conhecimento que o atleta tem da modalidade. Conforme Weineck (2003), iniciantes requerem um tempo de reação maior do que pessoas treinadas em todos os níveis de complexidade da tarefa realizada. Kokubu e Ando (2006), concluíram que o tempo de reação em atletas de voleibol é consideravelmente menor do que em não atletas em tarefas onde foram mensuradas respostas visuais e manuais. Atletas, aqueles mais experientes conseguem selecionar as dicas mais importantes quando comparados aos mais novos (LADEWIG, 2000). Autores como Rizola Neto (2003) e Schmidt e Wrisberg (2001) denominam esta capacidade de inteligência esportiva e consideram que esta é maior proporcionalmente a experiência e discernimento tático do esportista em questão.

Barbanti (1996) afirma que o tempo de reação pode ser influenciado de forma positiva pela concentração, atenção, aquecimento e por uma prévia tensão muscular; e de forma negativa pelo frio, pela fadiga, por fatores ambientais e por uma dificuldade de concentração. Weineck (2003) aponta para a importância da motivação e da concentração, o que pôde ser confirmado através de um estudo experimental onde foi comparado um primeiro bloco com dez tomadas de tempo de reação, com um último bloco da mesma atividade, também com dez aferições, sendo que o tempo do último bloco foi consideravelmente maior. 
Diversos fatores podem influenciar na velocidade de reação dos atletas. Dentre estes, podemos destacar a função que o atleta exerce na equipe. A especificidade é um dos princípios que regem o treinamento desportivo, estando relacionada à semelhança dos parâmetros fisiológicos, mecânicos e de controle motor dos exercícios utilizados nos treinos com aqueles encontrados em situação real de jogo (BARBANTI e ROCHA, 2004). Dentro de um processo metodológico de treinamento, o nível de especificidade das atividades deve evoluir proporcionalmente a evolução dos atletas (BARBANTI, 1996).

Atualmente, a maior parte das equipes de alto nível adota um sistema de jogo (5 x 1) em que todos os jogadores devem ser especialistas em determinadas funções. De acordo com a especificidade relacionada à sua responsabilidade, durante o próprio treinamento técnico e os jogos, o atleta é constantemente submetido a situações que podem ajudá-lo a obter respostas mais rápidas do que aqueles que normalmente não passam por tais situações, tornando determinados movimentos com um maior nível de automatismo (BOJIKIAN, 2005; BARBANTI e ROCHA, 2004). Um estudo recente de Maciel et al. (2009), corrobora esta afirmativa ao encontrar melhores escores do Tempo de Reação Motora em $(n=10)$ atletas Centrais quando comparados as outras funções específicas (Ponteiros $n=15$; Opostos $n=8$; Levantadores $n=8$ ). De acordo com os resultados encontrados, os atacantes centrais devem se preocupar principalmente com a marcação do bloqueio em todos os setores da rede, havendo a necessidade de se fazer uma "leitura" rápida da intenção de ataque do seu oponente para que ele possa, então, tentar neutralizá-la (BIZZOCHI, 2004).

Um outro fator recentemente apontado foi a taxa de metacognição dos atletas. Morales et al. (2009), definem a metacognição como o conhecimento que o sujeito tem sobre o seu próprio conhecimento, ou seja, o conhecimento dos próprios processos e produtos cognitivos que é percebido por nós para tomar uma atitude inteligente. Estes mesmos autores (2009), encontraram escores menores de Tempo de Reação Motora em (5) cinco atletas de voleibol com altos níveis de metacognição comparados aos atletas com os níveis inferiores, demonstrando que os níveis superiores de inteligência podem tornar o sujeito mais hábil no uso de estratégias eficazes de lidar, concomitantemente com maiores quantidades de informações no período menor de tempo. Esta "leitura" parece bastante de acordo com a existência de um "executivo central" inerente a toda a cognição, mais concretamente uma estrutura neurológica de ativação e atenção que asseguraria um fluxo organizado da informação na memória de trabalho.

\subsection{Perspectivas Futuras}

Devido a importância do Tempo de reação no voleibol tender a aumentar, proporcionalmente ao aumento da velocidade em que são desencadeadas as ações do jogo (MACIEL et al., 2009), acreditamos que mais pesquisas deverão ser realizadas a respeito do tema, esmiuçando detalhes específicos associados ao TR e o voleibol, o que pode vir a acrescentar mais qualidade aos treinamentos e ao desenvolvimento dos atletas de uma forma geral.

\section{CONSIDERAÇÕES FINAIS}

Os mecanismos fisiológicos responsáveis pela decodificação dos estímulos sensitivos em resposta motriz são os mesmos em vários tipos de esportes. A especificidade da mecânica dos gestos técnicos é que vai os diferenciar. Portanto, se torna importante o desenvolvimento do acervo motor e cognitivo inerente à modalidade, o que vai capacitar o praticante a efetuar respostas condizentes com os estímulos apresentados.

A evolução do voleibol exige que os detalhes que podem se tornar diferenciais para o máximo aproveitamento do potencial do atleta sejam conhecidos e revertidos em prol da melhoria do seu desempenho final, o que, por conseguinte, irá refletir na qualidade da sua equipe. Além da capacidade de realizar movimentos eficientes, uma das mais importantes características de um atleta habilidoso é 
a de ser proficiente na tomada de decisão sobre o que deve fazer em situações de jogo, especificamente quanto o tempo existente para encontrar a solução mais indicada é pequeno (Tempo de Reação).

De acordo com os relatos encontrados na literatura, o tempo de reação é uma variável que pode contribuir decisivamente no desempenho de diversos esportes, notadamente naqueles em que predomina a natureza "aberta" da maioria das ações, como é o caso do voleibol. Em razão da velocidade característica das ações do jogo, aliada a imprevisibilidade do ambiente, torna-se importante buscar desenvolver capacidades como a percepção, a antecipação e a diferenciação das situações práticas pelo atleta, com esses aspectos tendo uma importância por vezes superior à técnica requerida para a execução do fundamento.

\section{REFERÊNCIAS}

ANDRADE, L. C. G. O papel do tempo de reação nas ações táticas do voleibol. Dissertação de Mestrado apresentada ao programa de Pós-Graduação em Ciências Biológicas do Instituto de Pesquisa e Desenvolvimento da Universidade do Vale do Paraíba, São José dos Campos, 2004.

ARAÚJO, J. B. Voleibol moderno: sistema defensivo. Rio de Janeiro: Grupo Palestra Sport, 1994.

BARBANTI, V. J. Treinamento físico: bases científicas. 3ª ed. São Paulo: CLR Balieiro, 1996.

BARBANTI, V. J.; ROCHA, C. M. Uma análise dos fatores que influenciam o ataque no voleibol masculino de alto nível. Revista Brasileira de Educação Física e Esporte, v. 18, n. 4, p. 303-314, 2004.

BARCELOS, J. L.; MORALES, A. P.; MACIEL, R. N.; AZEVEDO, M. M. A.; SILVA, V. F. Tempo de prática: estudo comparativo do tempo de reação motriz entre jogadoras de voleibol. Fitness \& Performance Journal, v. 8, n. 2, p. 103-109, 2009.

BIZZOCHI, C. Voleibol: da Iniciação ao alto nível. São Paulo; Editora Manole, 2004.

BOJIKIAN, J. C. M. Voleibol atual: especialização ou universalidade. Revista do Vôlei, v.2, n. 3, p. 4$6,2005$.

BOMPA, T. O. Treinando atletas de desporto coletivo. Tradução: Juliana de Medeiros Ribeiro, Juliana Pinheiro de Souza e Silva. São Paulo: Phorte, 2005.

BOSSI, L. C. Musculação para o voleibol. São Paulo: Phorte, 2008.

BROWN, J. Improving reaction time. Sports Performance Journal. Março/2004. Disponível em www.athletesperformance.com, acesso em 10/06/2007.

CHAGAS, M. H.; LEITE, C. M. F.; UGRINOWITSCH, H.; BENDA, R. N.; MENZEL, H.-J.; SOUZA, P. R. C.; MOREIRA, E. A. Associação entre tempo de reação e de movimento em jogadores de futsal. Revista Brasileira de Educação Física e Esportes, v. 19, n.4, p. 269-275, 2005.

FONTANI, G.; LODI, L.; FELICI, A.; MIGLIORINI, S.; CORRADESCHI, F. Attentional in athletes of high and low experience engaged In different open skill sports. Perceptual and Motor Skills, v. 102, n. 3, p. 791-805, 2006.

FONTANI, G.; MAFFEI, D.; CAMELI, S.; POLIDORI, F. Reactivity and event-related potentials during attentional tests in athletes. European Journal of Applied Physiology and Occupational Physiology, v. 80, n. 4, p. 308-317, 1999. 
KOKUBU, M.; ANDO, S. Interference effects between saccadic and key-press reaction times of volleyball players and nonathletes. Perceptual and motor skills, v.103, n. 3, p. 709-716, 2006.

KRAMER, W. J. Treinamento de força para o esporte. Porto Alegre: Editora Artmed, 2004.

LADEWIG, I. A importância da atenção na aprendizagem de habilidades motoras. Revista Paulista de Educação Física, São Paulo, supl. 3, p. 62-71, 2000.

MACHADO, A. A. Voleibol: do aprender ao especializar. Rio de Janeiro: Guanabara Koogan, 2006.

MACHADO-PINHEIRO, W.; GAWRYSZEWSKI, L. G.; RIBEIRO do VALLE, L. E. Gap effect and reaction time distribution: simple vs choice manual responses. Brazilian Journal of Medical and Biology, v. 31, n. 10, p. 1313-1318, 1998.

MACIEL, R. N.; MORALES, A. P.; BARCELOS, J. L.; NUNES, W. J.; AZEVEDO, M. M. A.; SILVA, V. F. Relação entre tempo de reação e função específica em jogadores de voleibol. Fitness e Performance Journal, v. 8, n. 6, p. 395-399, 2009.

MAGGIL, R. A. Aprendizagem motora: conceitos e aplicações. São Paulo: Edgard Blucher, 2000.

MIYAMOTO, R. J.; MEIRA JÚNIOR, C. M. Tempo de reação e tempo das provas de 50 e 100 metros rasos do atletismo em federados e não federados. Revista Portuguesa de Ciências do Desporto, v. 4, n. 3,2004 .

MORALES, A. P.; AZEVEDO, M. M. A.; MACIEL, R. N.; BARCELOS, J. L.; ARÊAS NETO, N. T.; SILVA, V. F. Eficácia do processamento mental em jogadores de voleibol com níveis cognitivos diferenciados. Revista da Educação Física UEM, v. 20, n. 1, p. 43-50, 2009.

OLIVEIRA, M. A.; OLIVEIRA, C. A.; SOUZA, A. P. S. Medidas de tempo de reação simples em jogadores profissionais de voleibol. Revista Digital - Buenos Aires, Ano 10, N. 93 - Febrero de 2006, Disponível em www.efdeportes.com/, acesso em 23.07.07.

PORTELA, A. A influência da fadiga no tempo de reação em praticantes de escalada em rocha. Dissertação de mestrado em Ciências do Movimento Humano: Desenvolvimento e Aprendizagem Motora. Universidade Estadual de Santa Catarina, Florianópolis, 2005.

PRUDENCIO, V.; TUMELERO, S. Capacidades físicas e de treinamento para diferentes posições das praticantes da modalidade de voleibol. Revista Digital - Buenos Aires - Ano 10, n. 94, 2006. Disponível em: http://www.efdeportes.com/

RIZOLA NETO, A. Uma proposta de preparação para equipes jovens de voleibol feminino. Dissertação de mestrado apresentada ao programa de pós-graduação em Educação Física da UNICAMP, São Paulo. 2003.

ROCHA, C. M. O voleibol como um jogo esportivo coletivo: implicações para o treino. Revista do Vôlei, São Paulo, v. 2, n. 3, p. 44-48, 2005.

SANTOS, S.; TANI, G. Tempo de reação e a aprendizagem de uma tarefa de "timing" antecipatório em idosos. Revista Paulista de Educação Física, v. 9, n. 1, p. 51-62, 1995.

SCHMIDT, R. A.; WRISBERG, C. A. Aprendizagem e performance motora: Uma abordagem da aprendizagem baseada no problema. Porto Alegre: Editora Artmed, 2001.

SILVA, L. R. R. (editor). Desempenho esportivo: treinamento com crianças e adolescentes. São Paulo : Phorte, 2006. 
SILVA, L. R. R.; BOHME, M. T. S.; UEZU, R.; MASSA, M. A utilização de variáveis cineantropométricas no processo de detecção, seleção e promoção de talentos no voleibol. Revista Brasileira de Ciências e Movimento, Brasília, v. 11, n. 1, p. 69-76, jan. 2003.

STANGANELLI, L. C. R.; DOURADO, A. C.; ONCKEN, P.; MANÇAN, S. Caracterização da intensidade e volume das sessões de treino de voleibolista de alto rendimento. Revista Treinamento Desportivo, v. 7, n. 1, p. 06-14, 2006.

VOLCHAN, E.; PEREIRA, M. G.; OLIVEIRA, L.; VARGAS, C.; MOURÃO-MIRANDA, J.; VAGHETTI, C. A. O.; ROESLER, H.; ANDRADE, A. Tempo de reação simples auditivo e visual em surfistas com diferentes níveis de habilidade: comparação entre atletas profissionais, amadores e praticantes. Revista Brasileira de Medicina do Esporte. v. 13, n. 2, p. 81-85, 2007.

VOLCHAN, E.; PEREIRA, M. G.; OLIVEIRA, L.; VARGAS, C.; MOURÃO-MIRANDA, J.; AZEVEDO, T. M.; MACHADO-PINHEIRO, W.; PESSOA, L. Estímulos emocionais: processamento sensorial e respostas motoras. Revista Brasileira de Psiquiatria, v. 25, supl. II, pág. 29-32, 2003.

WAGNER, L. A. F. P.; SOUZA, C. H. M. Voleibol e mídia: uma sacada de ouro. Rio de Janeiro: Editora Damadá, 2007.

WEINECK, J. Treinamento Ideal. Tradução: Beatriz Maria Romano Carvalho. São Paulo: Manole, 2003. 